\title{
A practical multi-sensor activity recognition system for home-based care
}

\author{
Saisakul Chernbumroong ${ }^{\mathrm{a}}$, Shuang Cang ${ }^{\mathrm{b}}$, Hongnian $\mathrm{Yu}^{* a}$ \\ *corresponding authors \\ ${ }^{a}$ Faculty of Science and Technology, Bournemouth University, Fern Barrow, Poole, \\ Dorset, BH12 5BB \\ ${ }^{b}$ School of Tourism, Bournemouth University, Fern Barrow, Poole, Dorset, BH12 5BB
}

\begin{abstract}
To cope with the increasing number of ageing population, a type of care which can help prevent or postpone entry into institutional care is preferable. Activity recognition can be used for home-based care in order to help elderly people to remain at home as long as possible. This paper proposes a practical multi-sensor activity recognition system for home-based care utilizing on-body sensors. Seven types of sensors are investigated on their contributions toward activity classification. We collected a real data set through the experiments participated by a group of elderly people. Seven classification models are developed to explore contribution of each sensor. We conduct a comparison study of four feature selection techniques using the developed models and the collected data. The experimental results show our proposed system is superior to previous works achieving $97 \%$ accuracy. The study also demonstrates how the developed activity recognition model can be applied to promote a home-based care and enhance decision support system in health care.
\end{abstract}

Keywords: Multi-sensor activity recognition; home-based care; feature selection; classification; mutual information 


\section{Introduction}

The number of ageing population worldwide has increased rapidly. In 2010, there were 520 million people aged 65 years and over and is expected to increase to 1.9 billion people in 2050 [1]. Population ageing affects people in various aspects from society, politics to health care. Health care in particularly is greatly affected as people health deteriorate as they get older. These effects include high demand in long-term care, poor standard of care, and financial constraints in care expenditure. Different studies have been carried out with the aim of overcoming these effects. For example, an autonomous intelligent system was proposed in [2] for planing nurses' working time in order to provide effective care to alzheimer patients. The influencing factors that lead to initiate adoption of healthcare information systems was studied in [3]. The investigation was conducted in [4] to identify the level of autonomy-disability of an elderly people living in a nursing home for forecasting, planning and management of healthcare and social services.

Due to the effects of increasing older population, it is important to encourage preventive care to help prevent acute illness or delay entry into institutional care e.g. nursing homes, hospitals, etc. Examples of preventive care are ageing healthy and home-based care. Healthy ageing are such as eating healthy, regular exercising, regular health check-up, etc. Ageing healthily could extend longevity and reduce the possibility of acute serious illness. Another preventive care is to provide care at home such as health monitoring, activity monitoring, etc. Home-based care allows elderly people to be monitored seamlessly from their own homes allowing them to remain at home as long as possible. With current advance in sensors and technology, home-based care is possible and affordable for general population. 
Activity recognition is a part of home-based care. By manipulating and mining sensor data, current activity of a person can be determined. This information can be used to provide home monitoring, detect early sign of deterioration, provide a mean of assurance for family members, etc. Prior works in activity recognition are usually performed through visual sensing. However, this is not practical for elderly care application due to privacy issues resulting from the use of cameras. Due to this reason, a non-visual based activity recognition approach is more suitable. Recently, non-visual based activity recognition $[5,6]$ have been studied in an attempt of proposing a model that is practical and highly accurate.

Although these studies have demonstrated that activity recognition can benefit from combining information from multiple sensors, it is not yet clear how each of the sensors help in the detection of human activities. In this paper, we investigate seven types of sensors including accelerometer, temperature, altimeter, heart rate monitor, gyroscope, barometer and light sensor to understand how the loss of a particular sensor affects the classification accuracy and to which type of activity. We have collected a real data set from a group of elderly people performing a range of daily activities. This paper also studies several feature selection techniques and classification techniques in order to propose a practical activity recognition model. We compared our approach with other studies to demonstrate the superior in our model.

\section{Related works}

Based on sensors location, there are two main approaches in activity recognition i.e. infer activity from detected objects or changes in environment and infer activity from movement data. Object-based activity recog- 
nition requires sensors to be attached to numerous objects such as cups, toothbrush, tooth paste, spoon, etc. within homes. Sometimes sensors are also placed in environment for example, door switch [7], RFID [8], motion detectors [7] in rooms. This approach infers activity by observing the sequence of objects used or changes in environment. Although the approach can provide clear semantic toward activity recognition, it requires a large number of sensors installed in homes. Also, when there is a new object, a sensor must be tagged and the system needs to be updated. Problems related to uncertainty e.g. false start and fail to detect object can affect recognition performance. To address the problems, the approach which infers activity from movement data obtained from on-body sensors is adopted.

Human activity recognition based on on-body sensors has become popular due to the advance in sensor technology making sensors more accessible and affordable. A variety of on-body sensors have been explored such as accelerometer $[5,7,8,9,10,11]$, gyroscope $[6,11]$, temperature $[6,7,9]$, etc. Accelerometer is shown to be the most powerful sensor for activity recognition as it responds fast to movement change and can reflect the type of activity well [9]. A number of studies use several sensors attached to different parts of human body to increase recognition accuracy. Locations such as chest $[10,11]$, wrist $[5,6,7,11]$, thigh [10], waist [12], ankle [10, 11], etc. have been studied. For example, accelerometers were used on subjects' wrists, ankles and chest [11]. Inertial sensors were attached to chest, right thigh and left ankle to detect postures and transition activities [10]. However, attaching several sensors on body may decrease mobility or even obstruct daily activities routine. Also, these sensors may sometimes be perceived as stigmatisation. It is important, especially for elderly care applications, that the activity recognition system is practical with high performance. 
Taking aforementioned issues into consideration, some of studies proposed an activity recognition model based on a single location on human body [5]. Wrist is an ideal location for on-body sensors as it will not obstruct daily activity mobility. In this paper we consider the use of multiple sensor worn on wrist as we hypothesis that they will help yield more information necessary for activity recognition. Some studies were carried out based on wrist-worn multi-sensors. Multi-sensor wrist-worn equipment was used to detect walking, walking upstairs, walking downstairs, sitting and running activities [5]. The study showed that using a combination of accelerometer and light worn on wrist can produce good classification accuracy. Accelerometer, temperature sensor and altimeter worn on wrist were used to detect nine activities [6]. It showed that by combining accelerometer with temperature sensor and altimeter, classification accuracy is improved. Although the literatures indicated good results on the use of multiple sensors, it is not yet clear how each of the sensor help in activity classification. This prompt us to investigate how the loss of a particular sensor will affect the classification accuracy. Seven sensors have been selected including accelerometer, temperature, altimeter, gyroscope, barometer, light, and heart rate monitor. These sensors have been used in several prior works $[5,6,7,9,12,13,14,15]$. A study showed that by using gyroscope and magnetometer with accelerometer, the classification accuracy is increased by $17 \%$ [13]. Accelerometer and barometer were used to detect 11 children activities [12]. The results indicated improvement in accuracy after added barometer. Accelerometer and light sensor were used in [14] to detect seven office worker activities. A study showed that combining acceleration and heart rate improve accuracy of estimation of energy expenditure by $1.4 \%$ [15].

Based on these sensors, we propose an activity recognition model where 
we investigated several feature selection and classification techniques. As feature space becomes larger when several sensors are used, it is important that only relevant features for classification are selected. The feature selection technique usually measures the relationship between feature and the output e.g. by using information theory $[16,17,18]$, or by measuring the variable salient using Neural Network [6, 19], etc. For example, Minimal Redundancy Maximal Relevance (mRMR) [16] employs information theory to find a subset of features that have high mutual information between feature and output (maximal relevance) and low information among the selected features (minimal redundancy). Normalised Mutual Information Feature Selection (NMIFS) [17] also uses information theory. It claimed to be an enhancement over mRMR where normalised MI are used as a measurement of redundancy to reduce the bias of MI toward multi-valued features and also constraint value to be in $\left[\begin{array}{ll}0 & 1\end{array}\right]$ range. Feature Combination $(\mathrm{FC})$ technique uses neural network theory to perform feature selection. FC [6] takes into account a combination of feature to monitor network performance while features are added to the network. In this paper, we combine Clamping [19] with mRMR and NMIFS and compare it with other feature selection techniques including mRMR, NMIFS, and FC. Several classification algorithms such as Support Vector Machine (SVM) [6, 7, 12], Neural Network [6, 9, 11], Decision Tree $[8,9,12,5]$, etc. have been studied in human activity recognition. In this study, SVM, MLP, and RBF are investigated. 


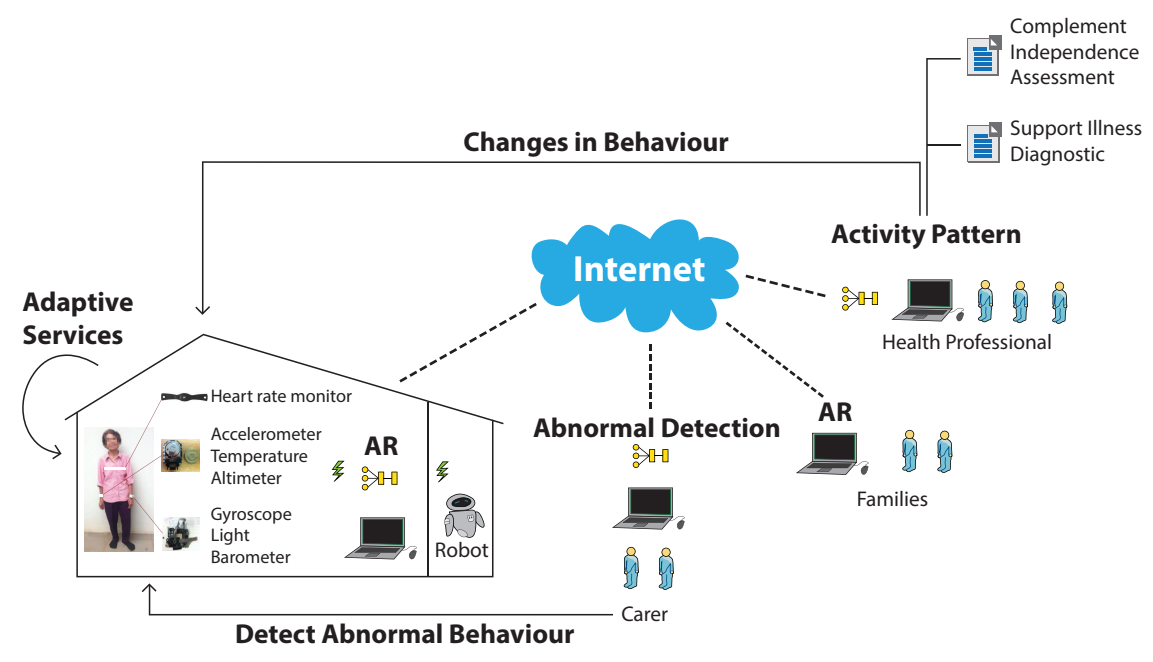

Figure 1: A practical multi-sensor activity recognition system for home-based care

\section{Methodology}

\subsection{Multi-sensor activity recognition system}

This section presents a practical multi-sensor activity recognition system shown in Figure 1 and describes how it can be used for home-based care. The elderly person wears sensors including accelerometer, temperature sensor, altimeter, gyroscope, light sensor, and barometer which are embedded on watch on their wrists and a heart rate monitor on their chests. The data from the sensor is continuously transmitted wirelessly through radio frequency to the PC in the elderly's home. The PC contains the activity recognition model (AR) which can recognise and detect daily activities of a user. The detected activity is perceived wirelessly by a companion robot who provides assistances or services based on current activity. For example, if the robot detects that the elderly person is exercising, it can play music or video related to that exercise. If the house is equipped with smart sensors, the detected activities can be used to provide information for adaptive services. For 
example, if it is detected that a user is sleeping, the light and the temperature can be adjusted to the suitable condition.

The detected activities can also be used by carer, health professionals, and families. To protect the privacy of the elderly person, the system will not send the raw sensor data over the network. The detected activities are encrypted when sent over the Internet. For carers, their systems will contain an activity abnormal detection model to detect abnormality of the elderly person. When the abnormal activity is detected, a carer can visit the elderly home and provide help. This will allow independence for both elderly person and carer, while maintain safety and good care when necessary. The families of the elderly person will also benefit from the system where they can use it to monitor them online anywhere and anytime to provide a peace of mind that their love ones are doing well. Health professionals will have access to the activity records. Their systems will contain a model which interpret each activity into activity patterns. They can use this as a complement to normal independent assessment and to support illness diagnostic. Also, if they detect any changes in behaviour, they could send a request to elderly person's system to retrieve a raw sensor data for further analysis or arrange a home or hospital visit for a check up on the elderly person.

Any sensor data sent from the elderly person must be encrypted and authorisation system must be installed and used whenever someone requested to access the data. Also, there must be a signed agreement on who can have access to what information and the elderly must give their consent prior the use of the system to ensure privacy and visibility. 


\subsection{Decision support system for health care}

This section describes how the proposed multi-sensor activity recognition can be used to enhance the decision support systems (DSS) for health care. Figure 2 shows the design of the DDS. The proposed method is used for classifying the complex sensor data into activities to generate a database of activity records over times. The data management is used for manage databases from several sources. The operations that the data management carry out includes organize, search, query, add, update, and delete databases. It also connects to the user interface management to provide interface for the users to perform operations with the databases. Besides the activity database, other databases related to health care information such as medical records, hospital resources, carer records, independence assessments, etc. are connected with the data management so that the DDS can cooperate several sources to make reliable decisions.

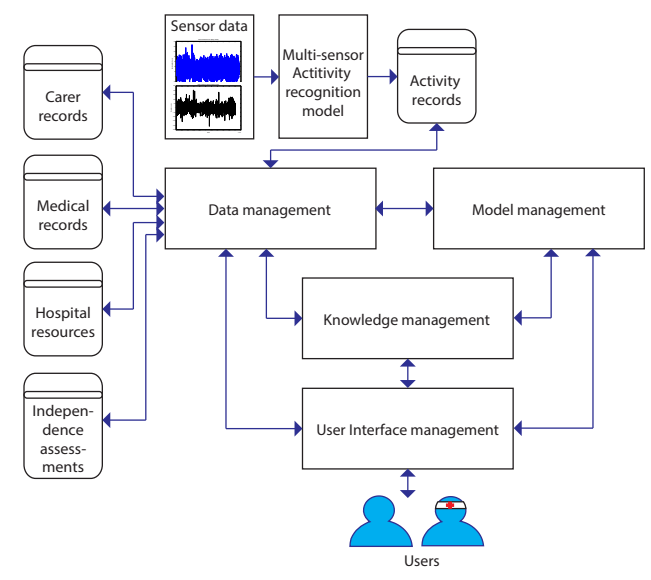

Figure 2: Decision support system for health car

The model management (MM) is used to manage models, select suitable models for different problems, execute the model, combine results from 
models. MM is connected to data management and UI to retrieve input data and to present outputs. The models are used to predict, simulate, etc. information. Example are such as a model to predict decline in daily activities, schedule the carer timetable, classify independence level, simulate utilities in hospital, etc.

In health care, experiences or expertise may be needed to make critical decisions. Therefore, the DDS contains the knowledge management (KM) which is used to store the knowledge resulting from the decision made by experts. The knowledge includes the process and/or information required to make decision by experts. KM consists of subsystems such as representation, validation, inference, and explanation of the knowledge.

The DDS contains the user interface management (UI) to manage different terminals for users to interact with the DDS. UI includes several interfaces suitable for different tasks and user groups. For example, the interface for management staffs should present overall result with graphical formats, while information of a particular task in details are presented to operational staffs. High usability is crucial aspect of the acceptance of DDS.

The DDS can be used to generate a monthly activity graph which shows the amount of each activity carried out in different months. This can be used to see the trend and detect changes in activities and support the decision whether to contact the person to come to the hospital and to which department or a home visit or whether further activity data should be requested from the patient. For example, if the graph shows the decline in walking over several months, this could suggest there is a problem with ambulating. This would help reduce the number of hospital visits, improve hospital resources utilization, and increase earlier detection rate.

The DDS can be used to support the decision on the type of carer is 
required for different patients. For example, if an activity record shows no decline or changes in activity pattern, carer may not be needed. If the activity record suggests the person may have problem with feeding, the carer who can provide assistance with feeding or cooking should be sent. Also, based on activity database, the DDS can build a model to predict when it is likely that the person will need a carer, so that the management of carer e.g. schedule, number of carer, etc. can be done effectively.

The activity record can be used for the assessment of independence. The DDS can use this to make a decision whether the carer is needed or predict when the carer will be needed in order to manage resources effectively. The activity database can be used as part of the other clinical decision support system to give more information to support the illness diagnostic or disease symptom. For example, if the activity record shows the patient has very little sleep per day could influence the decision of the specific sleeping disorder.

\subsection{System design justification}

The design of the system was based on the practicality factors for the assisted living system which was gathered from a questionnaire study. Questionnaires were distributed randomly at a major local hospital, nursing homes, general population in Stafford and the elderly club in Swansea to investigate senior adults perceptions on six assistive technologies and six factors regarding technology adoption. Descriptive statistics were used to analyze quantitative data. Qualitative data were analyzed by categorization techniques. The response rate of $74.7 \%$ was obtained of which 49 people were aged 60 years and over. The participants perceptions toward assistive technologies were positives except for video monitoring system. Privacy, cost, 
usability, reliability, functionality and misuse of technology were identified as concerns in assistive technologies adoption. The findings from this study indicate that privacy and cost are the most important issues which may affect technology adoption.

\subsection{Sensor characteristic and implementation}

We used the EZ-430 watch with integrated accelerometer, temperature sensor and altimeter on the CC430F6137 microcontroller with the MSP430 CPU from Texas Instrument (See Figure 3). The accelerometer measures 3 -axis acceleration between $\pm 2 \mathrm{G}\left(\mathrm{G}=9.81 \mathrm{~m} / \mathrm{s}^{2}\right)$ with sensitivity of 56 count/G. The pressure sensor can measure between $30-120 \mathrm{kPA}$ with $6 \mathrm{~Pa}$ resolution. The heart rate monitor chest strap is from BlueRobin. It has built-in $868 \mathrm{MHz}$ radio frequency which can transmit a range up to 800 meters. Temperature, altitude, and heart rate are logged in an $8 \mathrm{kB}$ flash on the watch. Acceleration is transmitted wirelessly to PC via application implemented on MatLab based on $868 \mathrm{MHz}$ radio frequency. Gyroscope, barometer, light sensor are implemented on Gadgeteer FEZ Cerberus board with $168 \mathrm{MHz}$ 32bit Cortex M4 processor. The gyroscope can measure up to $\pm 2000^{\circ} / \mathrm{s}$ with 14.375 LSBs per $\%$ s sensitivity. The barometer measures between 300 and $1100 \mathrm{hPa}$ absolute Pressure Range. The 2 GB SD card is used to log the data. The board was powered using an $800 \mathrm{mAh}$ power bank for a light weight application. The board was placed on the power bank which was placed on top of the wrist watch. Accelerometer and gyroscope are sampling at $33 \mathrm{~Hz}$, while the others are at $1 \mathrm{~Hz}$.

\subsection{Sensor location justification}

As the aim of this study is to propose a practical multi-sensor activity recognition system for home-based care, it was decided that the sensors 

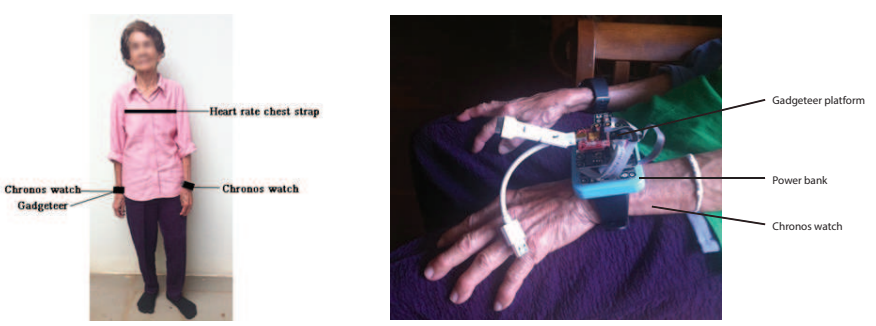

Figure 3: The location of the sensors. The gyroscope, barometer, and light sensor on Gadgeteer board are mounted over the Chronos watch. The participant wore two watches and a heart rate monitor on her chest.

should be worn at a users wrist. The justification of the system design on this work has been based on the literatures and innovative ideas. For example, the justification that using the accelerometer on the wrist is based on literatures that wrist is the optimum location for wearable sensor as it does not interrupt daily activities. Also, literatures indicate that it is possible to predict activities based on wrist-worn accelerometer.

However, due to hardware limitation, it was not possible to implements all the sensors on a single watch. Therefore, it was decided to separate the sensors between two wrists. We separate the sensors in a way that it should not interfere with the activity recognition. The sensors which are related to the movement i.e. accelerometer and gyroscope are worn on the dominant wrist in order to capture the activitys movement. Also, barometer and light sensors are also worn on the dominant wrist as they are parts of the Gadgeteer platform. The temperature sensor which captures the body temperature and altimeter are worn on the non-dominant wrist. In real application, we are expected to implement all the sensors into a single watch and will be worn on the dominant wrist of the elderly person. This location will not disrupt a user from performing an activity and/or cause discomfort 
Table 1: Participants characteristics for the Wearable-sensor Activity Data Set

\begin{tabular}{llllllllll}
\hline \multirow{2}{*}{ Gender } & \multicolumn{3}{c}{ Age (year) } & \multicolumn{3}{c}{ Weight $(\mathrm{Kg})}$. & \multicolumn{2}{c}{ Height $(\mathrm{m})}$. & \multicolumn{3}{c}{ BMI $\left(\mathrm{kg} / \mathrm{m}^{2}\right)$} \\
\cline { 2 - 10 } & Mean & Std. & Range & Mean & Std. & Mean & Std. & Mean & Std. \\
\hline female & 72.70 & 4.76 & 13.00 & 50.80 & 10.75 & 1.58 & 0.039 & 20.44 & 4.48 \\
male & 74.50 & 2.12 & 3.00 & 47.00 & 14.14 & 1.58 & 0.035 & 18.83 & 4.85 \\
all & 73.00 & 4.41 & 13.00 & 50.17 & 10.72 & 1.58 & 0.037 & 20.17 & 4.36 \\
\hline
\end{tabular}

in wearing sensors. The heart rate monitor needs to be worn on a users chest using a chest strap. Figure 3 shows the location of the sensors on a participant. Although the chest strap is made from elastic fabric, wearing the sensor for a continuous time might cause discomfort. The study will evaluate the trade-off between discomfort and the obtained accuracy.

\subsection{Data collection procedure}

The project was approved by the Faculty of Computing, Engineering and Technology Academic Ethics Team, Staffordshire University, UK. Before the data collection, all participants were asked about their age, gender, and health issues to evaluate their suitability for participation. We recruited 12 older participants and their characteristics are shown in Table 1. The number of participants is slightly larger than the average number of participants in activity recognition studies .

We studied 13 activities of daily living including brushing teeth, exercising, feeding, ironing, reading, scrubbing, sleeping, using stairs, sweeping, walking, washing dishes, watching TV and wiping. For exercise activity, the participants were asked to perform exercise using elastic stretching band. The participants were asked to carry out each activity for 10 minutes. They could perform the activity in any order. In total, 33.75 hours of activity data was recorded. We recorded 12 raw data including 3 axis of acceleration, 
heart rate, temperature, altitude, light, barometer temperature, barometer pressure, 3 axis of rotation. In total there are 64,084 patterns.

\subsection{Feature extraction}

It is difficult to built classification boundary directly from raw input, therefore suitable features need to be extracted or calculated from them. We first calculated the norm of both acceleration and rotation. There are 14 input data in total (12 raw data + acceleration norm + rotation norm). For each input, we calculated features from both time and frequency domains. These features include mean, standard deviation (STD), maximum, minimum, median, mode, kurtosis, skewness, intensity, difference, and rootmean-square (RMS), energy between $0.3-6 \mathrm{~Hz}$, entropy, key coefficient between $0.5-3 \mathrm{~Hz}$, correlations between each acceleration axis e.g. acc-X and acc-Y and correlations between each gyroscope axis e.g. gyro-X and gyro-Y. In total, 202 features were calculated.

As our feature space is large, it is important to carry out feature selection process. This process determines the smallest set of features while retaining the class discriminatory information. This will allow a classification model to be constructed effectively and reduce computational cost.

\subsection{Feature selection algorithms}

In this study, the following feature selection algorithms are investigated:

1. Minimal Redundancy Maximal Relevance (mRMR) [16]

It is based on the concept of the maximal statistical dependency criterion using MI which is used for defining the dependency between variables. Given two variables, $i, j$, the MI can be calculated as [20]: 


$$
I(i ; j)=\iint p(i, j) \log \frac{p(i, j)}{p(i) p(j)} \mathrm{d} i \mathrm{~d} j
$$

The mRMR technique employs the minimal redundancy maximal relevance criterion to achieve a maximal dependency condition. By combining mRMR and some subset selection algorithms e.g. forward selection, a subset of features, $S$, can be found by following steps:

(a) Given $S=\{\}$ where $S$ is a set of selected features and $F=$ $\left\{f_{1}, f_{2}, \ldots, f_{N}\right\}$ where $F$ is a set of $N$ features. Select the feature $f_{s}$ in $F$ which has the maximum mutual information between itself and output $C$ where $C=\left\{c_{1}, c_{3}, \ldots, c_{K}\right\}$ and $f_{s}=$ $\max _{f_{i} \in F} I\left(f_{i} ; C\right)$. Update $S$ and $F$.

$$
\begin{aligned}
& S=S \cup\left\{f_{s}\right\} \\
& F=F \backslash\left\{f_{s}\right\}
\end{aligned}
$$

(b) Select feature $f_{s}$ in $F$ which satisfies the following condition:

$$
\max _{f_{i} \in F}\left\{I\left(f_{i} ; C\right)-\frac{1}{|s|} \sum_{f_{j} \in S} I\left(f_{i} ; f_{j}\right)\right\}
$$

Update $S$ and $F$ using (1) and (2). Repeat step (b) until the desired number of features is obtained.

2. Normalised Mutual Information Feature Selection (NMIFS) [17] NMIFS is an enhancement of mRMR. Rather than using the average of MI as a measure of redundancy between feature and the subset of selected features as in mRMR, a normalised MI (NMI) is used:

$$
N M I(i ; j)=\frac{I(i ; j)}{\min \{H(i), H(j)\}}
$$


where $H()$ is the entropy function. Similar steps as mRMR is carried out, however the condition in step (b) is changed to:

$$
\max _{f_{i} \in F}\left\{I\left(f_{i} ; C\right)-\frac{1}{|s|} \sum_{f_{j} \in S} N M I\left(f_{i} ; f_{j}\right)\right\}
$$

3. Combination of mRMR, NMIFS and Clamping (COM)

We propose to combine feature rankings from mRMR, NMIFS and Clamping. The importance of the feature can be calculated as [19]:

$$
\operatorname{Im}\left(f_{i}\right)=1-\frac{g\left(F \mid f_{i}=\bar{f}_{i}\right)}{g(F)}
$$

where $g()$ is the generalized performance of the network.

Following steps are used to perform feature selection using Clamping:

(a) Calculate the importance of each feature $f_{i}$ using (3). A subset of features is selected according their importance.

$$
f_{s}=\max _{f_{i} \in F} \operatorname{Im}\left(f_{i}\right)
$$

(b) A subset of features is updated using (1) and (2).

These steps are repeated until the desired number of features is reached. The rankings from mRMR, NMIFS, and Clamping are combined using the Borda count. Given $N$ features, the highest score $N$ is given to the most important features and 1 to the least important features. The score is then combined for all the rankings from each feature selection techniques. The final ranking is obtained by sorting out the features in descending order (highest score for the most important feature).

4. Feature combination (FC) [6]

FC monitors the performance of the selected features so that the subset contains a suitable combination of features. First, the features are 
ranked using the Clamping technique. Then, the features are selected based on its importance using (4). Before a feature is added to $S$, an MLP network is constructed using $S$ and $F_{i}$ as input and if and only if $g\left(S \cup f_{i}\right) \geq g(S)$, then update $\mathrm{S}$ and F using (1) and (2). This is repeated until all features have been evaluated. All feature sets are combined using Borda count to obtain the final ranking.

\subsection{Classification algorithm}

After a suitable subset of features is identified, a classification model can be constructed. In this study, three classification algorithms are investigated. A brief description on these techniques is reviewed below. Given input $x_{i} \in \Re$ and output $o_{i} \in\{0,1, \ldots, K\}$.

1. Multi-Layer Perceptron neural network (MLP) [21]

MLP is capable of learning any nonlinear functions by adjusting the connection weights to minimize the error of the output. It utilizes the concept of connectionist where several input nodes are connected with several outputs nodes. These connections are associated with weights and the network output, and can be calculated as

$$
o_{i}=\phi\left(\sum_{i} w_{i} x_{i}\right)
$$

where $\phi$ is the activation or transfer function. MLP learns the classification error through the back propagation algorithm and minimizes that error by adjusting the weights $w_{i}$.

2. Radial Basis Function neural network (RBF) [21]

$\mathrm{RBF}$ is a network which uses the radial basis function as the activation function. For $N$ hidden neurons, the activation function is defined as: 


$$
f(x)=\sum_{i=1}^{N} w_{i} \varphi\left(\left\|x-c_{i}\right\|\right)
$$

where $c_{i}$ is the centre vector for neuron $i$ and $\varphi$ is a kernel function e.g. Gaussian, thin plate spline, etc.

3. Support Vector Machine (SVM) [22]

SVM projects inputs into a higher dimensional space so that non-linear data can be separated. It searches for hyperplane with a maximal margin to separate the data by solving the following optimization problem:

$$
\min _{w, b, \xi}\left[\frac{1}{2} w^{T} w+C \sum_{i=1}^{m} \xi_{i}\right]
$$

subject to:

$$
o_{i}\left(w^{T} f\left(x_{i}\right)+b\right) \geq 1-\xi_{i} ; \xi_{i} \geq 0
$$

The slack term $\xi_{i}$ is used to relax the constraints allowing misclassified examples. The associated cost parameter $C$ is used for penalizing $\xi_{i}$. $f()$ is a kernel function which transforms the input $x_{i}$ into a higher dimensional space. Common kernel functions are such as linear kernel, RBF kernel and polynomial kernel, etc. This study uses RBF kernel function $f\left(x_{i}\right)=\exp \left(-\frac{1}{\left(2 \sigma^{2}\right)}\left\|x_{i}-x_{j}\right\|^{2}\right)$ where $\sigma$ is the width of the Gaussian kernel. For K-class classification, $K$ binary classifiers are constructed and one-VS-all classification is applied.

\subsection{Statistical tests}

Statistical tests were employed to test if the difference in classification accuracy is significant. First, the data is tested against its normality using Shapiro-Wilk. If the data is normal distribution, Paired-sample T-test is 
used. Otherwise, Related-Sample Wilcoxon Signed Rank test is used. All statistics used were carried out at $95 \%$ confidence interval.

\section{Experimental results}

The collected data was pre-analysed and missing data was removed as it did not statistically affect the data set. Sweeping activity data was removed as after removing missing data this class only constitutes to $3 \%$ of the data set. Balanced class sampling is used to help build a more accurate decision boundary and make the model more robust to detect unknown input. Also, imbalanced class can impose problems such as error in interpreting classification results, and data from minority class may be treated as noise.

The data was pre-processing using weighted moving average and segmented at 3.88 seconds with $50 \%$ overlapping, resulting in 39,328 patterns. 202 feature were extracted as specified in Section 3.7. NaN and constant valued features were removed. Also to reduce the feature space, we calculated MI of each feature and decide a cut-off point at $3 \%$ of the maximum MI. Note that, MI is calculated on discretized data using 10 bins. As the result, there were 141 features. All experiments carried out in this study uses 10-fold cross validation where 8 folds are used for training, 1 for validation and 1 for testing. The data was randomly selected using uniform distribution. All experiments were repeated for 10 runs.

\subsection{Feature selection}

Firstly, features were ranked using the specified techniques mentioned in Section 3.8. The results from different runs are combined using the Borda count. Feature selection was performed using Neural Network. A multilayer perceptron with one hidden layer was used where the hidden node was set 
to $\alpha \times$ number of input. Experiments were carried out to determine the appropriate value of alpha and the number of epoch where trade-off between accuracy and training time were considered.

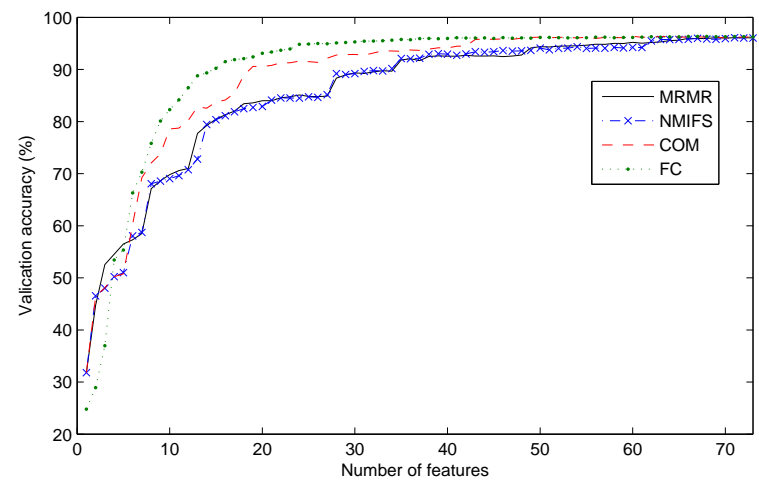

Figure 4: Validation accuracy on different feature selection techniques

The result of averaged validation accuracy is shown in Figure 4. From the graph, it can be seen that FC achieve the highest accuracy. We tested the hypothesis if the accuracy difference is significant. The data is not normal distribution and statistical tests indicated that the accuracy of FC is significant higher than other techniques $(p<0.05)$. COM is significant higher than mRMR and NMIFS $(p<0.05)$. The difference in accuracies of mRMR and NMIFS are not statistically significant $(p=0.315)$. To sum up, the performance of the feature selection techniques can be expressed as $F C>^{*} C O M>^{*} m R M R=N M I F S$ where $>^{*}$ indicates significantly better and $=$ indicates no significant difference at $95 \%$ confidence interval.

mRMR and NMIFS produced similar accuracy and selected similar set of features. The reason is that they are based on mutual information. This is evident in which mRMR and NMIFS produced similar ranking. When we investigated why these techniques cannot achieve higher accuracy, it was 
found that majority of the features selected at the beginning were from accelerometer and gyroscope only. Although features extracted from these sensors contain valuable information, when using the forward selection strategy this would lead to a selection of redundant features. mRMR and NMIFS only selects features from accelerometer, gyroscope and light sensor.

On the other hand, Clamping ranking selects features from a variety of sensors such as accelerometer, gyroscope, heart rate sensor, barometer, light, and altimeter (see Table 2). It can be seen that the result has considerably improved when COM is used. Besides accelerometer, gyroscope and light sensor, $\mathrm{COM}$ also selects features from barometer which means that this sensor provides valuable information for activity classification. Features selected from Clamping and FC are similar as FC is modified from Clamping technique. However, FC searches for only the subset of features which are complementing each other and reduce redundant features. FC clearly achieved better accuracy comparing to the other three techniques. However, according to the graph, the accuracies at the beginning are lower. Thus, in the case of data set with small number of features (less than 5), using mRMR should produce a better result. The truncation at 24 features was selected where the accuracy starts to remain constant.

\subsection{Classification}

The classification models were developed using classification algorithms as described in Section 3.9 with 24 selected features. Also, to demonstrate that the proposed method using more sensors can achieve better accuracy, we construct another model where 16 features from three sensors were used and classification is based on SVM [6]. From here, we shall refer this model as $S V M 16_{3 S}$. As the $S V M 16_{3 S}$ uses only 16 features, we also constructed 
Table 2: Features selected using different techniques

\begin{tabular}{|c|c|c|c|c|c|c|}
\hline Sensor & Data & MRMR & NMIFS & Clamping & $\mathrm{COM}$ & $\mathrm{FC}$ \\
\hline \multirow{4}{*}{ Accelerometer } & $\mathrm{X}-$ axis & - & - & RMS, mean & RMS & RMS, mean \\
\hline & $\mathrm{Y}$ - axis & $\begin{array}{l}\text { RMS, max, me- } \\
\text { dian, mode, key } \\
\text { coefficient, mean, } \\
\text { min }\end{array}$ & $\begin{array}{l}\text { Max, median, } \\
\text { mean, mode, min }\end{array}$ & $\begin{array}{l}\text { RMS, } \quad \text { max, } \\
\text { median, key co- } \\
\text { efficient, mode, } \\
\text { mean }\end{array}$ & $\begin{array}{l}\text { RMS, median, } \\
\text { mean, min, mode }\end{array}$ & $\begin{array}{l}\text { Max, median, } \\
\text { mean, min, mode, } \\
\text { RMS }\end{array}$ \\
\hline & $\mathrm{Z}$ - axis & $\begin{array}{l}\text { Min, median, } \\
\text { mode, mean }\end{array}$ & $\max$ & RMS, mean & $\begin{array}{l}\text { Mean, median, } \\
\text { min, mode }\end{array}$ & RMS, mean \\
\hline & $\sqrt{x^{2}+y^{2}+z^{2}}$ & $\begin{array}{l}\text { Intensity, } \max , \\
\text { median, mean, } \\
\text { RMS }\end{array}$ & $\begin{array}{l}\text { Intensity, RMS, } \\
\max \text {, mean }\end{array}$ & $\begin{array}{l}\text { Correlation } \mathrm{X}, \mathrm{Z} \text {, } \\
\max , \mathrm{RMS}\end{array}$ & $\begin{array}{l}\text { Max, intensity, } \\
\text { RMS, median, } \\
\text { mean }\end{array}$ & $\begin{array}{l}\text { Correlation X, Z, } \\
\max , \text { RMS }\end{array}$ \\
\hline Temperature & - & - & - & - & - & - \\
\hline Altimeter & Altitude & - & - & Min & - & Min \\
\hline $\begin{array}{ll}\text { Heart rate } \\
\text { monitor }\end{array}$ & Heart rate & - & - & - & - & Min \\
\hline Light & Light intensity & $\operatorname{Max}$ & $\operatorname{Max}$ & Max, min & $\begin{array}{l}\text { Max, RMS, mean, } \\
\text { median }\end{array}$ & Max, min \\
\hline \multirow[t]{2}{*}{ Barometer } & Temperature & - & - & $\begin{array}{l}\text { Max, median, } \\
\text { RMS, mean }\end{array}$ & Median, Max & $\begin{array}{l}\text { Max, median, } \\
\text { RMS }\end{array}$ \\
\hline & Pressure & - & - & Max, median & Max & Max, median \\
\hline \multirow{4}{*}{ Gyroscope } & $\mathrm{X}$ - axis & STD, RMS & STD, mode & - & STD & STD \\
\hline & $\mathrm{Y}$ - axis & - & - & - & - & - \\
\hline & $\mathrm{Z}$ - axis & $\begin{array}{l}\text { Std, RMS, inten- } \\
\text { sity }\end{array}$ & $\begin{array}{l}\text { Min, median, } \\
\text { mode, mean }\end{array}$ & - & - & - \\
\hline & $\sqrt{x^{2}+y^{2}+z^{2}}$ & $\begin{array}{l}\text { RMS, mean, me- } \\
\text { dian, std }\end{array}$ & $\begin{array}{l}\text { RMS, mean, me- } \\
\text { dian }\end{array}$ & Correlation X, Y & RMS & Correlation X, Y \\
\hline
\end{tabular}

classification models using truncation point at 16 features. The classification is performed using test data and the results are shown in Table 3. The notation of the model name is given by the algorithm, number of feature, and number of sensor. For example, $R B F 16_{7 S}$ represents the classification model using RBF with 16 features from 7 sensors.

The data is normal distribution and statistical test indicated that the differences between each model are statistically significant where $S V M 24_{7 S}$ > $^{*}$ $S V M 16_{7 S}>^{*} M L P 24_{7 S}>^{*} R B F 24_{7 S}>^{*} R B F 16_{7 S}>^{*} M L P 16_{7 S}>^{*}$ 
$S V M 16_{3 S}$ where $>^{*}$ indicates significantly better at $95 \%$ confidence interval. We also tested if there is a difference in accuracy when 16 and 24 features are used. The result indicated that using 24 features obtained statistically higher accuracy than using 16 features $(p<0.05)$.

Table 3: Test classification accuracy of each model

\begin{tabular}{lccccccc}
\hline Model & $S V M 16_{7 S}$ & $S V M 24_{7 S}$ & $M L P 16_{7 S}$ & $M L P 24_{7 S}$ & $R B F 16_{7 S}$ & $R B F 24_{7 S}$ & $S V M 16_{3 S}$ \\
\hline Mean & 96.9575 & 97.2040 & 94.8496 & 96.7349 & 95.3075 & 95.6734 & 85.4238 \\
Std. Error & 0.0349 & 0.0310 & 0.0421 & 0.0371 & 0.0413 & 0.0375 & 0.0672 \\
\hline
\end{tabular}

The results revealed that SVM is the best classification model among others. In general, the models can classify walking very well. However, they have difficulty in classifying feeding activity. The result shows that in our dataset SVM is superior to MLP and RBF. $S V M 24_{7 S}$ achieved the highest classification accuracy while $M L P 16_{7 S}$ achieved the lowest accuracy. When observing the F-score for each class, it was found that in general $S V M 24_{7 S}$ obtained the highest score, especially for exercise activity. $S V M 16_{7 S}$ achieved slightly better result in classifying brushing teeth and feeding than $S V M 24_{7 S}$. When observing precision and recall, it can be seen that $S V M 16_{7 S}$ achieved higher precision in washing dishes and watching TV comparing to $S V M 24_{7 S}$. While $S V M 24_{7 S}$ has higher sensitivity in obtaining these classes, $S V M 16_{7 S}$ makes prediction more accurately.

When examining classification algorithms using 24 features, we found that SVM has the highest F-score in most classes except feeding and reading where MLP is better. RBF has the lowest F-score in every class especially in feeding which is substantially lower. However, we found that RBF has comparable or even higher precision with SVM in some classes such as exercising, and reading. MLP has a comparable F-score with SVM in brushing 
teeth, washing dishes and watching TV. When examining at the models using 16 features (which is not the optimal number of features), SVM has the highest F-score in all classes. The F-score of RBF is higher than that of MLP in most classes except for brushing teeth and feeding.

The statistical results indicated that our models using 7 sensors obtained significant higher accuracy than the model based on 3 sensors regardless classification algorithms used. The improvement in accuracy is between $9.43 \%$ and $11.78 \%$. We then compare the F-score of each class between previous work and our SVM models. The results indicated that the proposed system achieved a higher F-score than $S V M 16_{3 S}$ model in all 12 activities (See Table 5). The F-score of all classes of the $S V M 24_{7 S}$ are higher than $S V M 16_{7 S}$ except for brushing teeth, feeding and wiping. When observing the confusion matrix of $S V M 24_{7 S}$ (See Table 4), we found that the model often confuses between feeding and brushing teeth, wiping and scrubbing, and walking and using stairs. Ironing and washing sometimes are also confused with feeding. It is observed that these activities have similar motion on the wrist.

To evaluate the trade-off between accuracy and the use of heart rate monitor, we performed classification without using the feature from the heart rate where we substitute the feature with the next best feature. The classification using MLP obtained $93.1020 \% \pm 0.5850 \%$. The data is normal distribution and the statistical test indicated that by removing heart rate feature, the classification accuracy is significantly lowered $(T=-28.993, p<0.05)$.

\subsection{Sensor contribution}

In this section, we consider how each sensor help with classification. We performed experiments to understand how the loss of a particular sensor 
Table 4: Confusion matrix of the $S V M 24_{7 S}$

\begin{tabular}{|c|c|c|c|c|c|c|c|c|c|c|c|c|}
\hline \multirow[t]{2}{*}{ Actual } & \multicolumn{12}{|c|}{ Predicted } \\
\hline & Brush & Exercise & Feed & Iron & Read & Scrub & Sleep & Stairs & Walk & Wash & Watch & Wipe \\
\hline Brush & 20246 & 29 & 280 & 65 & 53 & 20 & 30 & 85 & 0 & 101 & 47 & 44 \\
\hline Exercise & 40 & 20667 & 26 & 57 & 20 & 16 & 1 & 38 & 17 & 59 & 11 & 48 \\
\hline Feed & 289 & 35 & 19824 & 197 & 142 & 67 & 67 & 63 & 2 & 186 & 78 & 50 \\
\hline Iron & 91 & 69 & 162 & 20210 & 30 & 56 & 10 & 62 & 8 & 127 & 14 & 161 \\
\hline Read & 61 & 32 & 154 & 101 & 20463 & 14 & 25 & 20 & 5 & 40 & 68 & 17 \\
\hline Scrub & 9 & 23 & 34 & 58 & 6 & 20549 & 8 & 29 & 4 & 38 & 40 & 202 \\
\hline Sleep & 65 & 9 & 70 & 24 & 21 & 37 & 20526 & 124 & 2 & 28 & 26 & 68 \\
\hline Stairs & 86 & 37 & 96 & 38 & 14 & 55 & 44 & 20498 & 99 & 22 & 30 & 29 \\
\hline Walk & 0 & 33 & 3 & 8 & 6 & 38 & 8 & 153 & 20670 & 6 & 0 & 27 \\
\hline Wash & 78 & 28 & 208 & 123 & 54 & 66 & 19 & 34 & 19 & 20278 & 18 & 75 \\
\hline Watch & 13 & 6 & 19 & 8 & 55 & 6 & 30 & 72 & 6 & 20 & 20742 & 23 \\
\hline Wipe & 43 & 52 & 17 & 112 & 25 & 164 & 21 & 48 & 20 & 195 & 29 & 20274 \\
\hline
\end{tabular}

Table 5: F-score comparison between models based on 3 sensors and 7 sensors

\begin{tabular}{llrrrrrrrrrrr}
\hline Model & Brush & Exercise & Feed & Iron & Read & Scrub & Sleep & Stairs & Walk & Wash & Watch & Wipe \\
\hline SVM $16_{3 S}$ & 0.7684 & 0.8670 & 0.7575 & 0.8214 & 0.8496 & 0.8615 & 0.9478 & 0.8771 & 0.9530 & 0.8069 & 0.9398 & 0.8055 \\
$S V M 16_{7 S}$ & $\mathbf{0 . 9 6 4 9}$ & 0.9725 & $\mathbf{0 . 9 4 7 1}$ & 0.9580 & 0.9748 & 0.9685 & 0.9814 & 0.9661 & 0.9883 & 0.9631 & 0.9852 & $\mathbf{0 . 9 6 5 3}$ \\
SV $M 24_{7 S}$ & 0.9636 & $\mathbf{0 . 9 8 3 7}$ & 0.9464 & $\mathbf{0 . 9 6 2 4}$ & $\mathbf{0 . 9 7 7 0}$ & $\mathbf{0 . 9 7 6 5}$ & $\mathbf{0 . 9 8 2 4}$ & $\mathbf{0 . 9 6 9 8}$ & $\mathbf{0 . 9 8 8 9}$ & $\mathbf{0 . 9 6 3 3}$ & $\mathbf{0 . 9 8 5 3}$ & 0.9650 \\
\hline
\end{tabular}

affects the classification accuracy and to which activity. To control the experiment, top features (based on MI) of each sensor were selected to use in the classification. The selected features are maximum acceleration Y-axis, maximum heart rate, maximum barometric pressure, maximum light intensity, RMS gyro magnitude, minimum temperature, and minimum altitude.

Firstly, we generated a classification model (called base model) which uses all sensors. We constructed the next model by removing one sensor. For example, model 1 used all sensor except accelerometer. Model 2 used all sensor except heart rate sensor. In total, 8 models were built. The 
notation of the model is given by $\mathrm{M}$ followed by the name of the removed sensor e.g. $M_{A c c}$ represents model which does not use accelerometer. The classification was performed using MLP and the number of hidden nodes is twice the number of input. Table 6 shows mean accuracy of the model when a particular sensor is not used. The test of normality indicated that model $M_{\text {Light }}$ is not normal distribution, thus we employed Wilcoxon Signed Ranks to test the effect of the loss of a sensor. The statistical results indicate that there is a statistical significant different between the base model and all the other models $(p<0.05)$. Based on the reduced accuracy, the contribution of the sensor can be ranked from the highest to the lowest as accelerometer, gyroscope, light sensor, barometer, heart rate sensor, temperature sensor, and altimeter, respectively. We examined the F-score of each class of each model (See Table 7). The model which does not include accelerometer has affect on several activities including brushing teeth, feeding, ironing, reading, scrubbing, walking, and wiping. The effect on the absent of light sensor is on sleeping, stairs, and washing dishes activities. The model whithout a gyroscope sensor has effects on exercise and watching TV activity.

\begin{tabular}{llrr}
\multicolumn{2}{c}{ Table 6: } & The effect of the loss of a particular sensor \\
\hline Model & Missing sensor & Accuracy (\%) & Std. Deviation \\
\hline Base model & None & 65.1913 & 1.4354 \\
$M_{\text {Acc }}$ & Accelerometer & 50.0933 & 1.4140 \\
$M_{H R}$ & Heart rate sensor & 62.0873 & 1.2548 \\
$M_{\text {Baro }}$ & Barometer & 60.7004 & 1.2010 \\
$M_{\text {Light }}$ & Light sensor & 57.6663 & 1.1589 \\
$M_{\text {Gyro }}$ & Gyroscope & 55.8540 & 1.4780 \\
$M_{\text {Temp }}$ & Temperature sensor & 62.2528 & 1.1885 \\
$M_{\text {Alt }}$ & Altimeter & 62.8056 & 1.1016 \\
\hline \multicolumn{4}{c}{}
\end{tabular}


Table 7: F-score of each model

\begin{tabular}{lrrrrrrrrrrrrr}
\hline Model & Brush & Exercise & Feed & Iron & Read & Scrub & Sleep & Stairs & Walk & Wash & Watch & Wipe \\
\hline Base model & 0.6771 & 0.5818 & 0.5506 & 0.5856 & 0.5549 & 0.7140 & 0.7382 & 0.7144 & 0.7809 & 0.5191 & 0.7088 & 0.6683 \\
$M_{\text {Acc }}$ & 0.5036 & 0.4438 & 0.4239 & 0.3715 & 0.4271 & 0.5025 & 0.6579 & 0.6437 & 0.3858 & 0.4307 & 0.6048 & 0.5325 \\
$M_{H R}$ & 0.6493 & 0.5382 & 0.5393 & 0.5797 & 0.5122 & 0.6826 & 0.6995 & 0.6995 & 0.7725 & 0.4459 & 0.6652 & 0.6229 \\
$M_{\text {Baro }}$ & 0.6406 & 0.5494 & 0.5397 & 0.5483 & 0.4824 & 0.6771 & 0.6456 & 0.6639 & 0.7596 & 0.4715 & 0.6500 & 0.6211 \\
$M_{\text {Light }}$ & 0.5688 & 0.5639 & 0.4673 & 0.5640 & 0.5062 & 0.6843 & 0.5994 & 0.5354 & 0.7428 & 0.3973 & 0.6193 & 0.6035 \\
$M_{\text {Gyro }}$ & 0.5995 & 0.3807 & 0.4841 & 0.5147 & 0.4879 & 0.5878 & 0.6676 & 0.6402 & 0.7286 & 0.4489 & 0.4838 & 0.6304 \\
$M_{\text {Temp }}$ & 0.6544 & 0.5410 & 0.5405 & 0.5644 & 0.5197 & 0.6968 & 0.7204 & 0.6885 & 0.7541 & 0.4542 & 0.6816 & 0.6155 \\
$M_{\text {Alt }}$ & 0.6583 & 0.5541 & 0.5417 & 0.5624 & 0.5157 & 0.7094 & 0.7033 & 0.6885 & 0.7645 & 0.4883 & 0.6814 & 0.6359 \\
\hline
\end{tabular}

\section{Discussion}

In this paper we developed several models to investigate the absent of a particular sensor. It was found that each sensor has a significant contribution toward the classification accuracy in general. This means that each sensor has given specific information which is useful for activity classification. The results also show that accelerometer is the most important sensor since the classification accuracy has significantly dropped when the sensor is not used. However, missing this sensor does not strongly affect the detection of sleeping. This is due to the fact that this activity is not involved in much movement. On the other hand, missing the light sensor has significantly affected sleeping detection. This suggests the model uses information from the light sensor to detect sleeping activity. Similarly, stairs activity is also affected by missing light intensity information. When observing the plot of the maximum light intensity of these two classes, it is found that, unlike other classes, the data from these two activities are rather clustered. Therefore, missing this information affects the classification of these two classes. The absent of gyroscope has effect on exercise and watching TV activities. This shows that although the $M_{\text {Gyro }}$ model contains accelerometer feature, it is not enough to detect these activities. RMS of gyro magnitude signifi- 
cantly helps classify these activities. Although the results demonstrate that each of the seven sensors are important, these models are constructed based on only one feature from each sensor. It is possible that when a model is developed with more number of features, information from a particular sensor could be substituted by the other features from other sensor as well. In fact, in the proposed model, temperature sensors are not selected.

Comparing with $S V M 16_{3 S}$, the results suggest that the addition of heart rate sensor, barometer, gyroscope and light sensor improve classification accuracy. This means that they provide valuable information for classification of the activities studied. The results of the study provide suggestion on possible sensors for other activity classification systems. Also, these sensors except for heart rate monitor are used on a users wrist will allow practical applications of activity recognition for home-based care. The results show that our proposed system achieves statistically better performance.

The results show that combining heart rate with other sensor significantly improves classification accuracy. Nevertheless, the classification accuracy without using heart rate is still high comparing to $S V M 16_{3 S}$. This suggests that it is possible to use only wrist worn sensors to maintain its practicality and better accuracy can be achieved.

Table 5 indicates that our model achieves comparable or in some activities higher than previous studies. Also, our approach only requires sensor worn on wrist and chest. We also show that even we remove the heart rate sensor, high accuracy can be achieved. This is an important aspect for a practical application in elder care. The system which is not intrusive or perceived as stigmatization can be easily accepted by the elderly.

Another objective of this study is to compare the performance of 4 feature selection techniques. Our results suggest that FC is the most appro- 
Table 8: Accuracy comparison between previous works and the proposed system

\begin{tabular}{|c|c|c|c|c|c|c|c|c|c|}
\hline & \#activity & Sensor location & Brush teeth & Feed & Iron & Sleep & Stairs & Walk & Average \\
\hline$S V M 24_{7 S}$ & 12 & Wrist,chest & 96.36 & 94.64 & 96.24 & 98.24 & 97.39 & 98.65 & 97.20 \\
\hline [11] & 12 & wrists, ankles, chest & - & 89.50 & - & 89.20 & 90.80 & 88.20 & 91.3 \\
\hline$[7]$ & 7 & Body, environment & 64.30 & 97.80 & - & 93.90 & - & 95.00 & 86.20 \\
\hline$[8]$ & - & Wrist, objects & - & - & 97.94 & 92.66 & - & 84.36 & - \\
\hline [9] & 7 & On-body & - & - & 87.00 & - & 79.00 & 86.00 & $82-86$ \\
\hline$[5]$ & 6 & Wrist & - & - & - & - & $>90$ & - & 87.10 \\
\hline [10] & 12 & Chest, thigh, left ankle & - & - & - & 95.4 & - & 98.1 & 91.4 \\
\hline
\end{tabular}

priate technique for our application. FC can select a more diversity set of features comparing to other techniques. It monitors the performance of a subset of features along the selection to make sure that redundant features are not selected. However, according to the FC algorithm, redundant features may still be selected at earlier stage and we suggest that post checking should be added to remove any redundant feature after selection. mRMR and NMIFS only measure the redundancy between 2 variables which was shown not enough to reduce the overlapped features. The result of this study implies that the technique which can select a subset of features with the lowest feature redundancy is the most optimum technique.

\section{Conclusion}

We have proposed a practical multi-sensor activity recognition system for home-based care and evaluated it through the real data we collected. We investigated seven types of sensors including accelerometer, temperature, altimeter, heart rate monitor, barometer, light sensor, and gyroscope on how it helps classification accuracy and to which types of activity. In general we found accelerometer to be the most important sensor. We also found that 
maximum light intensity can be useful for detecting sleeping, stairs, washing dishes activities. RMS of gyro magnitude can help in classifying exercise and watching TV activities. Although we found that all the sensors provide important information toward classification, when larger features of sensors are available, a particular sensor could be omitted.

We compared the results with previous method which only used three sensors and the results show that the additional four sensors help improve activity classification accuracy. We achieve $97.2040 \%$ accuracy using six sensors. The study also demonstrates how the developed activity recognition model can be applied for home-based care and DDS for health care. The study also investigated 4 feature selection techniques including mRMR, NMIFS, COM and FC. The results indicate that FC can select the optimum set of features as it can select features from diverse sensors which helps reduce feature redundancy. We suggest improvement on this technique by adding a post feature check to remove redundant feature which may be selected during earlier stage. Also, further investigation on the proposed model in a natural setting is recommended.

\section{Acknowledgement}

We thank Mrs Pan Ayumak and Mrs Juntip Tubtimsri, the representatives of Watket elderly club, Chiang Mai, Thailand and Mr Somchit Chernbumroong for their support on data collection.

\section{References}

1. UN, . World population prospects: The 2012 revision. 2012. URL: http://esa.un.org/unpd/wpp/index.htm. 
2. Corchado, J.M., Bajo, J., de Paz, Y., Tapia, D.I.. Intelligent environment for monitoring alzheimer patients, agent technology for health care. Decision Support Systems 2008;44(2):382 - 396.

3. Yang, Z., Kankanhalli, A., Ng, B.Y., Lim, J.T.Y.. Analyzing the enabling factors for the organizational decision to adopt healthcare information systems. Decision Support Systems 2013;55(3):764 - 776.

4. Combes, C., Azema, J.. Clustering using principal component analysis applied to autonomydisability of elderly people. Decision Support Systems 2013;55(2):578 - 586.

5. Maurer, U., Rowe, A., Smailagic, A., Siewiorek, D.. Location and activity recognition using ewatch: A wearable sensor platform. Ambient Intelligence in Everyday Life 2006;:86-102.

6. Chernbumroong, S., Cang, S., Atkins, A., Yu, H.. Elderly activities recognition and classification for applications in assisted living. Expert Systems with Applications 2013;40(5):1662 - 1674.

7. Fleury, A., Vacher, M., Noury, N.. Svm-based multimodal classification of activities of daily living in health smart homes: Sensors, algorithms, and first experimental results. Information Technology in Biomedicine, IEEE Transactions on 2010;14(2):274-283. ID: 1.

8. Hong, Y.J., Kim, I.J., Ahn, S.C., Kim, H.G.. Mobile health monitoring system based on activity recognition using accelerometer. Simulation Modelling Practice and Theory 2010;18(4):446 - 455.

9. Parkka, J., Ermes, M., Korpipaa, P., Mantyjarvi, J., Peltola, J., Korhonen, I.. Activity classification using realistic data from wearable 
sensors. Information Technology in Biomedicine, IEEE Transactions on 2006;10(1):119-128.

10. Trabelsi, D., Mohammed, S., Chamroukhi, F., Oukhellou, L., Amirat, Y.. An unsupervised approach for automatic activity recognition based on hidden markov model regression. Automation Science and Engineering, IEEE Transactions on 2013;10(3):829-835.

11. Wang, Z., Jiang, M., Hu, Y., Li, H.. An incremental learning method based on probabilistic neural networks and adjustable fuzzy clustering for human activity recognition by using wearable sensors. Information Technology in Biomedicine, IEEE Transactions on 2012;16(4):691-699.

12. Nam, Y., Park, J.. Child activity recognition based on cooperative fusion model of a triaxial accelerometer and a barometric pressure sensor. Biomedical and Health Informatics, IEEE Journal of 2013;17(2):420426.

13. Gjoreski, H., Gams, M.. Activity/posture recognition using wearable sensors placed on different body locations. Proceeding of signal and image processing and applications 2011;.

14. Lombriser, C., Bharatula, N.B., Roggen, D., Tröster, G.. On-body activity recognition in a dynamic sensor network. In: Proceedings of the ICST 2nd international conference on Body area networks; BodyNets '07. 2007, p. 17:1-17:6.

15. Munguia Tapia, E.. Using machine learning for real-time activity recognition and estimation of energy expenditure. Ph.D. thesis; Massachusetts Institute of Technology; 2008. 
16. Peng, H., Long, F., Ding, C.. Feature selection based on mutual information: criteria of max-dependency, max-relevance, and minredundancy. IEEE Transactions on Pattern Analysis and Machine Intelligence 2005;27:1226-1238.

17. Estevez, P., Tesmer, M., Perez, C., Zurada, J.. Normalized mutual information feature selection. Neural Networks, IEEE Transactions on 2009;20(2):189-201.

18. Cang, S., Yu, H.. Mutual information based input feature selection for classification problems. Decision Support Systems 2012;54(1):691 698.

19. Wang, W., Jones, P., Partridge, D.. Assessing the impact of input features in a feedforward neural network. Neural Computing $\mathfrak{E}^{3}$ Applications 2000;9(2):101-112.

20. Shannon, C.E.. A mathematical theory of communication. SIGMOBILE Mob Comput Commun Rev 2001;5(1):3-55.

21. Bishop, C.M.. Neural Networks for Pattern Recognition. New York, NY, USA: Oxford University Press, Inc.; 1995.

22. Chang, C.C., Lin, C.J.. LIBSVM: A library for support vector machines. ACM Transactions on Intelligent Systems and Technology 2011; $2: 27: 1-27: 27$. 